\title{
BMC Pharmacology and Toxicology reviewer acknowledgement 2014
}

\author{
Christopher Morrey
}

\section{Contributing reviewers}

The editors of BMC Pharmacology and Toxicology would like to thank all of our reviewers who have contributed to the journal in Volume 15 (2014).

\begin{tabular}{lll} 
Peter Aduloju & George Bayliss & Daniel Cardinali \\
Nigeria & USA & Argentina \\
Gustav Akk & Vikhyat Bebarta & Michael Carvan \\
USA & USA & USA \\
Catrin Albrecht & Getenet Beyene & Jose Castillo-Mancilla \\
Germany & Ethiopia & USA \\
Adam Algren & Sagnik Bhattacharyya & Luigi Cattel \\
USA & UK & Italy \\
Hisham Aljadhey & Marialuisa Bocchino & Sarah Cavanaugh \\
Saudi Arabia & Italy & USA \\
Maria Almeida & Jalel Boukadida & Thomas Chan \\
Portugal & Tunisia & Hong Kong \\
Lamya Alnaim & Peter Bruegger & Debabrata Chanda \\
Saudi Arabia & Switzerland & India \\
Nicolas Andre & Thomas Brunner & Mario Chiong \\
France & Germany & Chile \\
Usman Bala & Miran Brvar & Cristiana Cipriani \\
Malaysia & Slovenia & Italy \\
Alfred Balch & Martin Burtscher & Francesco Clementi \\
USA & Austria & Italy \\
Douglas Ball & Sarah Campbell & Michael Coughtrie \\
Philippines & USA & Canada \\
Desmond Bannon & Domenico Capone & Guillaume Darrasse-Jèze \\
USA & Italy & France \\
\hline
\end{tabular}

Correspondence: christopher.morrey@biomedcentral.com

BioMed Central, Floor 6, 236 Gray's Inn Road, London, WC1X 8HB, United

Kingdom

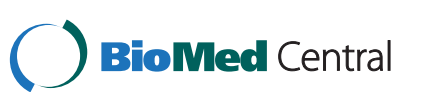




\begin{tabular}{|c|c|c|}
\hline Clara De Palma & David Haas & Barbara Kaplan \\
\hline Italy & USA & USA \\
\hline $\begin{array}{l}\text { Fabrizio De Ponti } \\
\text { Italy }\end{array}$ & $\begin{array}{l}\text { Katja Marja Hakkarainen } \\
\text { Sweden }\end{array}$ & $\begin{array}{l}\text { Michalis Karamouzis } \\
\text { Greece }\end{array}$ \\
\hline $\begin{array}{l}\text { Francesco Giuseppe De Rosa } \\
\text { Italy }\end{array}$ & $\begin{array}{l}\text { David Hardman } \\
\text { USA }\end{array}$ & $\begin{array}{l}\text { Marlies Karsch-Voelk } \\
\text { Germany }\end{array}$ \\
\hline $\begin{array}{l}\text { Philip Debruyne } \\
\text { Belgium }\end{array}$ & $\begin{array}{l}\text { Thomas Hartung } \\
\text { USA }\end{array}$ & $\begin{array}{l}\text { Jun-Ichi Kawabe } \\
\text { Japan }\end{array}$ \\
\hline $\begin{array}{l}\text { Domenico Delfino } \\
\text { Italy }\end{array}$ & $\begin{array}{l}\text { Yuya Hayashi } \\
\text { Denmark }\end{array}$ & $\begin{array}{l}\text { Ulrike Kemmerling } \\
\text { Chile }\end{array}$ \\
\hline $\begin{array}{l}\text { Shalinee Dhayal } \\
\text { UK }\end{array}$ & $\begin{array}{l}\text { Mai Helmy } \\
\text { Egypt }\end{array}$ & $\begin{array}{l}\text { Tahir Khan } \\
\text { Malaysia }\end{array}$ \\
\hline $\begin{array}{l}\text { Robert Dickinson } \\
\text { UK }\end{array}$ & $\begin{array}{l}\text { Robert Hendrickson } \\
\text { USA }\end{array}$ & $\begin{array}{l}\text { Alexander Koch } \\
\text { Germany }\end{array}$ \\
\hline $\begin{array}{l}\text { Eric Dietrich } \\
\text { USA }\end{array}$ & $\begin{array}{l}\text { Francisco Hernández-Luis } \\
\text { Mexico }\end{array}$ & $\begin{array}{l}\text { Shyam Kottilil } \\
\text { USA }\end{array}$ \\
\hline $\begin{array}{l}\text { Kelly Dooley } \\
\text { USA }\end{array}$ & $\begin{array}{l}\text { Samuel Heyman } \\
\text { Israel }\end{array}$ & $\begin{array}{l}\text { Monica Lamberti } \\
\text { Italy }\end{array}$ \\
\hline $\begin{array}{l}\text { Vangelis Economou } \\
\text { Greece }\end{array}$ & $\begin{array}{l}\text { Lisa Hines } \\
\text { USA }\end{array}$ & $\begin{array}{l}\text { Cornelia Landersdorfer } \\
\text { Australia }\end{array}$ \\
\hline $\begin{array}{l}\text { Kamal El-Atrebi } \\
\text { Egypt }\end{array}$ & $\begin{array}{l}\text { Nguyen Quynh Hoa } \\
\text { Vietnam }\end{array}$ & $\begin{array}{l}\text { Vincenzo Lariccia } \\
\text { Italy }\end{array}$ \\
\hline $\begin{array}{l}\text { Ibrahim Elgendy } \\
\text { Egypt }\end{array}$ & $\begin{array}{l}\text { Corinne Hohl } \\
\text { Canada }\end{array}$ & $\begin{array}{l}\text { Lawrence Lash } \\
\text { USA }\end{array}$ \\
\hline $\begin{array}{l}\text { Joseph Fadare } \\
\text { Nigeria }\end{array}$ & $\begin{array}{l}\text { Hidehito Horinouchi } \\
\text { Japan }\end{array}$ & $\begin{array}{l}\text { Ping-Ing Lee } \\
\text { Taiwan }\end{array}$ \\
\hline $\begin{array}{l}\text { Rodrigo Franco } \\
\text { USA }\end{array}$ & $\begin{array}{l}\text { Lan Huang } \\
\text { China }\end{array}$ & $\begin{array}{l}\text { Zhoumeng Lin } \\
\text { USA }\end{array}$ \\
\hline $\begin{array}{l}\text { Casper Franssen } \\
\text { Netherlands }\end{array}$ & $\begin{array}{l}\text { Marilyn Huestis } \\
\text { USA }\end{array}$ & $\begin{array}{l}\text { Kenneth Litwak } \\
\text { USA }\end{array}$ \\
\hline $\begin{array}{l}\text { Yubin Ge } \\
\text { USA }\end{array}$ & $\begin{array}{l}\text { Shehnaz Ilyas } \\
\text { India }\end{array}$ & $\begin{array}{l}\text { Juan Carlos Lopez-Delgado } \\
\text { Spain }\end{array}$ \\
\hline $\begin{array}{l}\text { Alex Georgakilas } \\
\text { Greece }\end{array}$ & $\begin{array}{l}\text { Shazia Jamshed } \\
\text { Malaysia }\end{array}$ & $\begin{array}{l}\text { Rodrigo López-Muñoz } \\
\text { Chile }\end{array}$ \\
\hline $\begin{array}{l}\text { Kheirollah Gholami } \\
\text { Iran }\end{array}$ & $\begin{array}{l}\text { Piotr Janicki } \\
\text { USA }\end{array}$ & $\begin{array}{l}\text { Henrik Lövborg } \\
\text { Sweden }\end{array}$ \\
\hline $\begin{array}{l}\text { Joel Goodman } \\
\text { USA }\end{array}$ & $\begin{array}{l}\text { Zhenquan Jia } \\
\text { USA }\end{array}$ & $\begin{array}{l}\text { Zuxun Lu } \\
\text { China }\end{array}$ \\
\hline $\begin{array}{l}\text { Andrew Lofts Gray } \\
\text { South Africa }\end{array}$ & $\begin{array}{l}\text { Paul Jurasz } \\
\text { Canada }\end{array}$ & $\begin{array}{l}\text { Yongjun Luo } \\
\text { China }\end{array}$ \\
\hline $\begin{array}{l}\text { Kristin Grimsrud } \\
\text { USA }\end{array}$ & $\begin{array}{l}\text { Keizo Kanasaki } \\
\text { Japan }\end{array}$ & $\begin{array}{l}\text { Simona Magi } \\
\text { Italy }\end{array}$ \\
\hline $\begin{array}{l}\text { Tyler Gums } \\
\text { USA }\end{array}$ & $\begin{array}{l}\text { Gopal Kannan } \\
\text { India }\end{array}$ & $\begin{array}{l}\text { Tadeusz Malinski } \\
\text { USA }\end{array}$ \\
\hline
\end{tabular}


Wimolnut Manheng

USA

Alexandre Manirakiza

Central African Republic

Chiedza Maponga

USA

David Martinez

Spain

Ulrike Mende

USA

Christine Metz

USA

Martin C Michel

Netherlands

Kathryn Momary

USA

Ichiro Morioka

Japan

Danina Muntean

Romania

Chris Murgatroyd

Germany

Celso Vataru Nakamura

Brazil

Eiji Nemoto

Japan

Detlef Neumann

Germany

Seiji Niho

Japan

Stefania Nobili

Italy

Alfredo Nunziata

Italy

Fernando Palhano

Brazil

Giorgio Palu

Italy

Vasileios Papavasileiou

Greece

Kevin Park

UK

Davide Pastorelli

Italy
Martin Payne

UK

Angelo Peli

Italy

Napoleon Perez-Farinos

Spain

Andrei Pernambuco

Brazil

Jesus Pintor

Spain

Antonius Plagge

UK

Maria Cristina Da Silva Pranchevicius Brazil

Matiram Pun

Nepal

Guoqing Qian

USA

Marek Radomski

Ireland

Shashi Ramaiah

USA

Emanuel Raschi

Italy

Marcia Ratner

USA

Cavan Reilly

USA

David Roberts

UK

Roberto Rodriguez-Roisin

Spain

Staffan Rosenborg

Sweden

Maurizio Rossini

Italy

Douglas Ruden

USA

Mitsue Saito

Japan

Yoshiro Saito

Japan

Laura Schnackenberg

USA
Angela Slitt

USA

Gabriele Stangl

Germany

Maciej Stepnik

Poland

Gabriele Stocco

Italy

Chris Stockmann

USA

Robert Matthew Strother

New Zealand

Ami Fazlin Syed Mohamed

Malaysia

Giovanni Tarantino

Italy

Pierre Teira

Canada

Maria Thurston

USA

Bennard Van Ravenzwaay Germany

Andras Varro

Hungary

Raja Venkatasubramanian

USA

Charles Venuto

USA

Andrew Vernon

USA

Chris Vulpe

USA

Shigeo Wakabayashi

Japan

Niansong Wang

China

Jian Wang

China

Henrik Watz

Germany

Matthew Whittaker

USA

Bin Yang

UK 
Yanqing Yi

Canada

Ebru Yildirim

Turkey

Tian Yu

USA

\section{Anna Zaghini}

Italy

Nathalie Zgheib

Lebanon

Naixia Zhang

China
Liming Zhou

China

Mario Zoratti

Italy 